\title{
Measuring the Mach number of the universe via the Sunyaev-Zel'dovich effect
}

\author{
F. Atrio-Barandela ${ }^{1}$, A. Kashlinsky ${ }^{2}$ \\ and J.P. Mücket ${ }^{3}$ \\ ${ }^{1}$ Física Teórica. Facultad de Ciencias. 37008 Salamanca, Spain email: atrio@usal.es \\ ${ }^{2}$ SSAI, Code 685, Goddard Space Flight Center, Greenbelt, MD 20771 \\ ${ }^{3}$ Astrophysikalisches Institut Potsdam, D-14482, Potsdam
}

\begin{abstract}
The cross-correlating cosmic microwave background (CMB) fluctuations caused by the Sunyaev-Zel'dovich effect from observed clusters of galaxies with their redshifts can be used to measure the mean squared cluster peculiar velocity with an error $\sigma_{C_{S}^{2}} \simeq(300 \mathrm{~km} / \mathrm{s})^{2}$. This can be done around $z>0.3$ with clusters of flux above $200 \mathrm{mJy}$ which will be detected by PLANCK, coupled with high resolution microwave images to eliminate the cosmological part of the CMB fluctuations. The latter can be achieved with observations by the planned ALMA array or the NSF South Pole telescope and other surveys. By measuring the rms peculiar velocity of clusters and their bulk flow in, e.g., 4 spheres of $\sim 100 h^{-1} \mathrm{Mpc}$ at $z=0.3$, we could have a direct measurement of the matter density $0.21<\Omega_{m}<0.47$ at $95 \%$ confidence level.
\end{abstract}

\section{Introduction}

The Mach number $\mathcal{M}$ characterizes the coldness of the velocity field. It was defined as $\mathcal{M}(r)=V_{\text {bulk }}(r) / C_{S}$ by Ostriker \& Suto (1990) who introduce it as a test of cosmological models. The bulk flow component is the mean peculiar velocity over a region of characteristic scale $r$ and $C_{S}$ is the rms peculiar velocity in the reference frame of the flow. The cosmic Mach number effectively measures the slope of the power spectrum up to the scales corresponding to the size of the region and can be used to constrain cosmological models for structure formation. Hot gas in moving clusters produces redshift independent cosmic microwave background (CMB) anisotropies (Sunyaev \& Zel'dovich, 1972, hereafter SZ). The thermal component (TSZ) is usually larger than the kinetic term (KSZ), but has a distinct spectral signature and can be removed using multifrequency observations, allowing the determination of the cluster peculiar velocities (Holzapfel et al. 1997; Mauskopf et al. 2000; LaRoque et al. 2002).

At present, the error bars on peculiar velocities of individual clusters, measured from KSZ $\left(\sigma_{v_{P}}\right)$ are much larger than the estimated velocities $v_{P}$, preventing reliable measurement of the Mach number using CMB data alone. Measurements of the CMB temperature of clusters and of their redshift can be combined to determine $\mathcal{M}$ in some specific configurations. The bulk flow velocity can be evaluated directly from the KSZ signal by adding the velocity of all clusters in the sample (Benson et al. 2003) with uncertainty:

$$
\sigma_{V_{\mathrm{bulk}}} \simeq \frac{\sigma_{v_{P}}}{\sqrt{N_{c l}}} \sim 100\left(\frac{\sigma_{v_{P}}}{1000 \mathrm{~km} / \mathrm{s}}\right)\left(\frac{N_{c l}}{100}\right)^{-1 / 2} \mathrm{~km} / \mathrm{s} .
$$

The rms peculiar velocity along the line of sight of clusters located on a thin shell in redshift space can be reliably measured by cross-correlating the temperature field at cluster locations with their measured redshifts. The cross-correlation gives (Atrio-Barandela, 

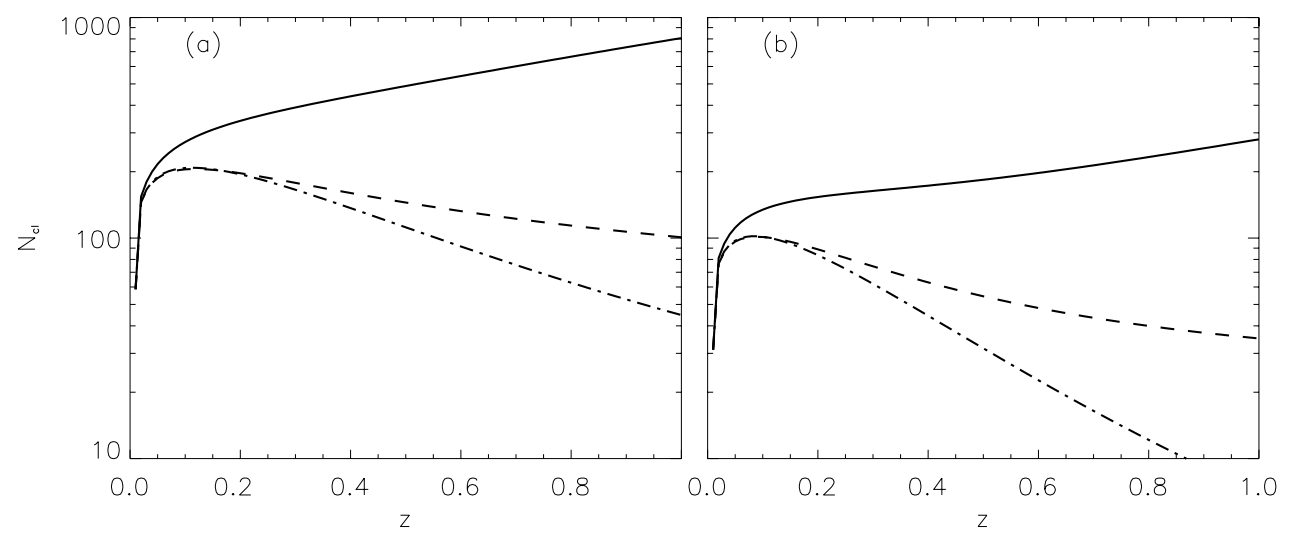

Figure 1. (a) Number of Clusters for three different XLF evolutionary histories: no evolution (solid line), dashed to $(A, B)=(-3,0)$ and dot-dashed to $(-1,-2)$. The detection limit is 200 mJy. (b) Same as before, but the threshold limit is $400 \mathrm{mJy}$.

Kashlinsky \& Mücket, 2004):

$$
\frac{\langle c \delta T \cdot c \delta z\rangle}{T_{o}\langle\tau\rangle}=C_{S}^{2} \pm \bar{\sigma}_{v_{P}} \frac{c \Delta z}{\sqrt{12 N_{c l}}} \pm\left\langle v_{p}\right\rangle \frac{\left(c \Delta z+2 C_{S}\right)}{\sqrt{12}} .
$$

The error bar of $C_{S}$ depends linearly on $\Delta z$. To reduce it we compute $C_{S}$ in shells with a small spread in redshift. For this geometry, the dominant contribution is

$$
\sigma_{C_{S}^{2}}=(300 \mathrm{~km} / \mathrm{s})^{2}\left(\frac{\bar{\sigma}_{v_{P}}}{1000 \mathrm{~km} / \mathrm{s}}\right)\left(\frac{\Delta z}{0.01}\right)\left(\frac{100}{N_{c l}}\right)^{1 / 2} .
$$

This represents an improvement of one order of magnitude on the current error bar of $C_{S}$.

\section{Observational prospects}

Observations of clusters to measure their SZ amplitude are already (almost) routinely carried out by several telescopes (e.g. Carlstrom et al. 2002) and exponential progress is expected with the construction of the ALMA array and the NSF funded South Pole microwave telescope. For the purpose of this project, high resolution observations of large samples of clusters with well measured redshifts are required. Blind surveys searching for clusters in large areas of the sky, like the cluster catalog expected to be obtained from the full sky CMB observations of the upcoming PLANCK ESA satellite mission will be most relevant. Most clusters detected by PLANCK will be unresolved. Those needed for computing the Mach number will have to be re-observed at high resolution with ALMA, where its high frequency coverage and its small projected noise of $\sim 2 \mu \mathrm{K}$ rms after one hour of integration time for a beam of $1^{\prime}$, could be very useful to subtract foreground and set the CMB contribution to its minimum.

To compute the number of clusters that will be detected by PLANCK, we modeled the X-ray Luminosity Function (XLF) as an evolving Schechter function: $\phi(L, z)=\phi_{o}(1+$ $z)^{A} L^{-\alpha} \exp \left(L / L_{*}\right)$, with $L_{*}=L_{*, 0}(1+z)^{B}$, where $A, B$ are two evolutionary parameters, and $L_{*}, \alpha$ are the local XLF values. We took $L_{*}=9.3 \times 10^{44} h^{-2} \mathrm{ergs} \mathrm{s}^{-1}$ and $\phi_{o}=2.44 \times 10^{-7} h^{3} \mathrm{Mpc}^{-3}$ (Ebeling et al. 1997). In Fig. 1, solid lines correspond to no redshift evolution $(A, B)=(0,0)$, dashed to $(-3,0)$ and dot-dashed to $(-1,-2)$, obtained 

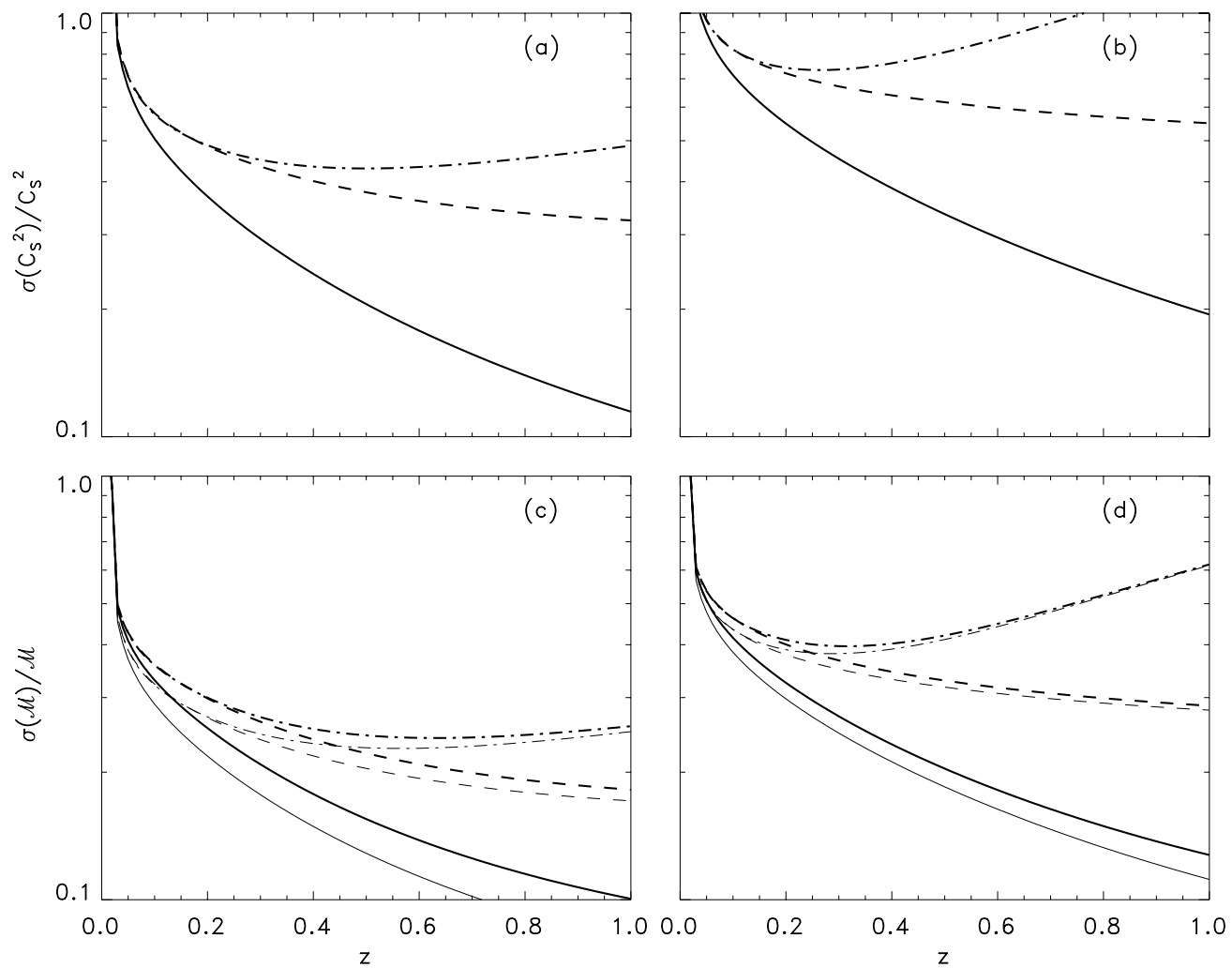

Figure 2. (a) Relative error of the sound speed for a flux detection limit of $200 \mathrm{mJy}$ and (b) $400 \mathrm{mJy}$. (c) Relative error on the Mach number, for the same evolution histories as before. In every pair, the upper line corresponds to bulk flows on a sphere of $100 h^{-1} \mathrm{Mpc}$, and the lower line to a sphere of $150 h^{-1} \mathrm{Mpc}$. The flux limit is $200 \mathrm{mJy}$ and (d) is $400 \mathrm{mJy}$.

from the analysis of the ROSAT Deep Cluster Survey and the Extended Medium Sensitivity Survey (EMSS), respectively. In (a) we show the number of clusters that produce a change in flux of $200 \mathrm{mJy}$ relative to the mean flux of the CMB, and in (b) those producing a change of $400 \mathrm{mJy}$. For the $200 \mathrm{mJy}$ limit (Fig. 1a) the RDCS evolution parameters predict about 14000 clusters out to $z=1$, and for the $400 \mathrm{mJy}$, about 6000 . Those numbers were 12000 and 4000 for the EMSS evolution parameters,

In Fig. 2(a,b) we show the relative errors of $C_{S}^{2}$ vs $z$. Lines correspond to the same evolution models as before. Spheres of radius $\simeq 100 h^{-1} \mathrm{Mpc}$ will contain very few clusters in blind all-sky surveys such as PLANCK cluster catalog, and another type of survey is required. Romer et al. (2001) argue that an XMM serendipitous cluster survey of about $\simeq 800 \mathrm{deg}^{2}$ will detect more than 8000 clusters ranging from poor to very rich systems. In Fig. 2c and 2d we plot the error on the Mach number assuming that the sound speed was computed in shells of PLANCK detected clusters and the bulk velocity is computed using a top hat window on spheres of 100 (thick lines) and $150 h^{-1} \mathrm{Mpc}$ (thin lines) radii.

Fig. 2c corresponds to PLANCK detecting all clusters with flux above $200 \mathrm{mJy}$ and (d) to $400 \mathrm{mJy}$. We also considered $\bar{\sigma}_{v_{P}}=1000 \mathrm{~km} \mathrm{~s}^{-1}$, although now the bulk velocity and sound speed are computed over different samples. 


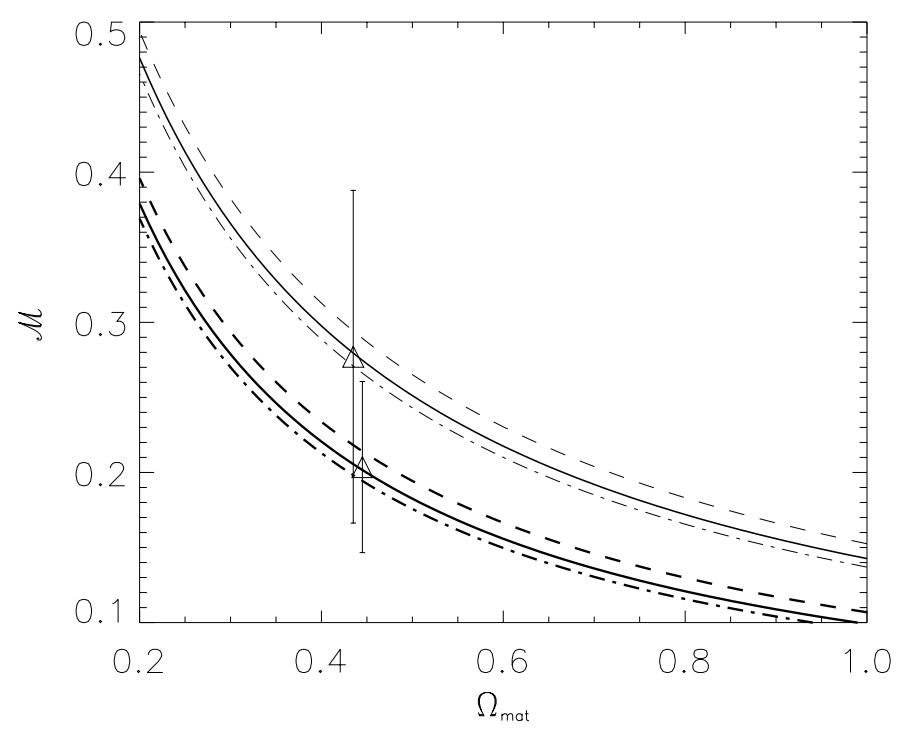

Figure 3. Mach number estimated by measuring bulk flows on spheres of 100 (upper set) or $150 h^{-1} \mathrm{Mpc}$ (lower set). Dashed, solid and dot-dashed lines correspond to a spectral index $n=0.95,1.0$ and 1.03, in agreement with WMAP results. The triangles show the prediction for the WMAP concordance model. The $1 \sigma$ error was computed assuming that there were 4 independent measurements of the bulk flow at that redshift. They are slightly shifted for a better display.

The Mach number can be used to estimate the mean matter density. For this purpose, let us assume that at redshift $z \simeq 0.3$ we have identified about $\sim 100$ PLANCK clusters with fluxes above $200 \mathrm{mJy}$ in a shell of $\Delta z=0.01$. The sample is used to compute $C_{S}$ at that $z$. We also assume that the clusters identified by XMM in a sphere of radius $100 h^{-1} \mathrm{Mpc}$ centered at $z$ have data of similar quality. The Mach number would be the ratio of the bulk velocity in the sphere to the sound speed in the shell.

Fig. 3 plots $\mathcal{M}$ vs matter density for flat models with cosmological constant, $\Omega_{\Lambda}+$ $\Omega_{m}=1$, and for three different values for the primordial spectral index $n=0.95,1,1.03$ shown as dashed, solid and dot-dashed lines (all other cosmological parameters were chosen from the WMAP measurements, Bennett et al. 2003, Spergel et al. 2003). The matter power spectrum was normalized to $\sigma_{8}=0.8$. The upper set of lines corresponds to bulk flows on spheres of $100 h^{-1} \mathrm{Mpc}$ and the lower set to $150 h^{-1} \mathrm{Mpc}$ radius. For $\Omega_{m}=0.3$ the triangle shows the expected Mach number and its 1- $\sigma$ error bars. We assumed that the cluster XLF evolves with RDCS parameters. If the bulk flow is measured for 4 independent spheres of $100 h^{-1} \mathrm{Mpc}$ radius, we can estimate $0.25(0.21) \leqslant \Omega_{m} \leqslant 0.37(0.47)$ at the 68 (95)\% confidence level. Our method is insensitive to a running spectral index, quintessence or tilt: variations on these cosmological parameters lead to differences in the Mach number much smaller than $\sigma_{\mathcal{M}}$. In this respect, our measurement of the matter density would be robust.

\section{Conclusions}

We presented a new method to measure the cosmic sound speed of clusters of galaxies. Assuming that all clusters with SZ flux larger than $200 \mathrm{mJy}$ will be identified by the 
PLANCK mission, and that the XRLF evolution in the Rosat Deep Cluster Survey is representative of the overall cluster evolution, the relative error would be $\sigma_{C_{S}^{2}} / C_{S}^{2} \sim 0.3$. If the XMM Serendipitous Cluster Survey detects all clusters above $k T_{X} \stackrel{s}{=} 4 \mathrm{keV}$, by combining measurements of bulk flows in spheres of different sizes with the cosmic sound speed measured in shells at the same redshift, we can estimate the Cosmic Mach number with relative accuracy $\sigma_{\mathcal{M}} / \mathcal{M} \geqslant 0.2$. This accuracy can be achieved with high resolution microwave images in order to reduce the intrinsic CMB anisotropy with respect to the kinematic SZ signal, and is within the reach of the currently planned South Pole and ALMA telescopes. The Cosmic Mach number we can determine by our method is directly related to the matter density, and it can determine $0.21 \leqslant \Omega_{m} \leqslant 0.47$ at the $95 \%$ confidence level. This result, being independent of other mass density measurements, would provide an important self consistency check.

\section{References}

Atrio-Barandela, F. et al. 2004, ApJ, 601, L111

Bennett, C.L. et.al., 2003, ApJS, 148, 1

Benson, B.A. et al. 2003, astro-ph/0303510

Carlstrom, J. E., Holder, G.P. \& Reese, E.D. 2002, ARA\&A, 40, 643

Ebeling, H. et al. 1997, ApJ, 479, L101

Holzapfel, W.L. et al. 1997, ApJ, 481, 35

LaRoque, S.L. et al. 2002, astro-ph/0204134

Mauskopf, P.D. et al. 2000, ApJ, 538, 505

Ostriker, J.P. \& Suto, Y. 1990, ApJ, 348, 378

Romer, A.K. et al. 2001, ApJ, 547, 594

Spergel, D.N. et.al., 2003, ApJS, 148, 175

Sunyaev, R.A. \& Zel'dovich, Ya. B. 1972, Comments Astrophys. Space Phys., 4, 173 